\title{
Multi-point Fundraising and Distribution via Blockchain
}

\author{
Abdullah Omar Abdul Kareem Alassaf ${ }^{1}$, Fakhrul Hazman Yusoff ${ }^{2}$ \\ Faculty of Computer Science and Mathematical Sciences \\ Universiti Teknologi MARA \\ Shah Alam, Malaysia
}

\begin{abstract}
Trust and transparency are significant facets that are much esteemed by charitable organizations in achieving their mission and encouraging donations from the public. However, after many high-profile scandals, the faith in charities is questionable, heralding the need for an increased level of transparency among such organizations. Fortunately, leveraging Blockchain technology in charities' systems could help to rebuild the integrity of these organizations. This study aims to raise the level of integrity showcased by charities by creating a multi-point fundraising approach using smart contracts. The proposed system offers a transparent fundraising platform through its integration of charity organization evaluators. Various steps were deployed to satisfy the intended target. Firstly, the study investigated the potentials of Blockchain in improving the level of transparency. Secondly, a probing process was undertaken to choose a suitable platform as a server-side in the system. This process involved garnering salient features in Blockchain platforms based on the proposed system requirements. After the probing process, a Decision Support System (DSS) was utilized to investigate the most suitable Blockchain platform. Results garnered proved that the Ethereum platform is best for the proposed system.
\end{abstract}

Keywords-Blockchain; smart contract; transparency; charity

\section{INTRODUCTION}

The desire to help and to be of service to others is the nature of human behaviour. Donations to charities are one of the ways people help each other and this is positively reflected on the community. Charities play an essential role in fields like education, healthcare, and other social services [1]. In addition, all religions preach and encourage philanthropy, for instance, Islam makes it obligatory and calls it Zakat [2].

Trust and confidence are fundamental for charitable bodies to achieve their mission in getting donors and donations [3]. Higher trust levels could increase the amount of donations received by these charities [1]. However, after many highprofile scandals, charities have been criticized for misusing donated money [4], and donors have begun to lose trust in charities; hence calls for more transparency have increased [5].

For instance, recent events and evidences show that public trust and confidence in charities in the UK has been damaged. As a result, the activities of individual charities could be limited, and the sustainability of the whole sector reduced [6].
The following figure is a survey has been done in North Ireland to explore the views on the charity sector; Fig. 1 shows a question related to transparency in charities.

Fig. 1 shows that $92 \%$ of the people, who answered the survey, agreed on that charities should be transparent about how public donations are spent, and how important it is that charities must demonstrate how they benefit the public, while $40 \%$ know how to find information about how charities are run. Also, only $32 \%$ know where they can find information about how charities are spending their money [7].

According to the facts and findings mentioned above, transparency is a crucial element in charity sector. Yet, it is one of the biggest vulnerabilities in the existing charity systems, as addressed in many studies [1], [3], [5], and [7]. Therefore, it should be enhanced.

With the advent of blockchain, many studies suggested that blockchain has a great potential to benefit various sectors and industries such as education [8] and travel [9].

Similarly, Blockchain is reputedly able to solve the transparency issues in charity systems [10]. Thus, leveraging blockchain technology in charity systems could help in rebuilding the image of charity. Hence, studies and researches are investigating different approaches to take the advantages of this technology.

This study aims to tackle the transparency issue. Therefore, a donation system will be implemented to promote transparency in charitable organizations' campaigns by using smart contracts to hold donors' donations till it gets verified based on multi-point model to ensure that donations reach those who need help. The deployed smart contracts store on a suitable blockchain platform which will be selected after identifying the important features in blockchain platforms to get the ideal platform for charity systems. The system offers a transparent environment for all parties including charitable organizations, donors, recipients, and charity evaluator organizations. As well as, all transactions will be made through this system will be accessible and traceable for the public. 


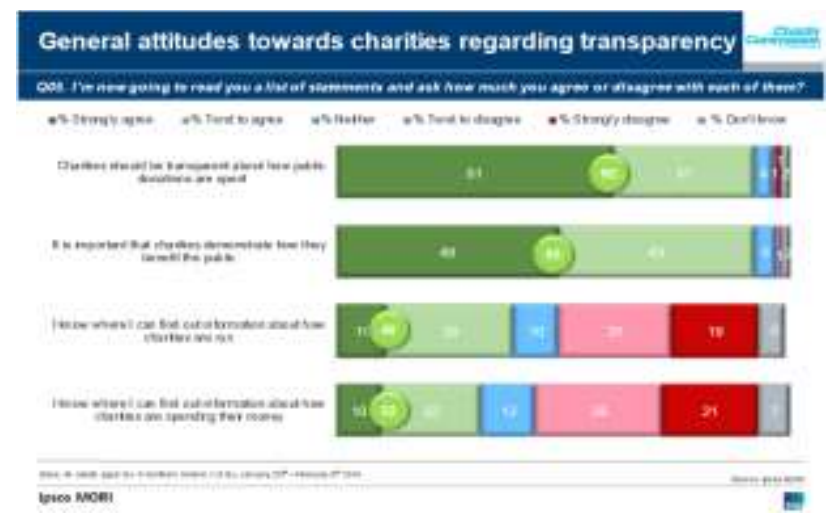

Fig. 1. General Attitudes towards Charities Regarding Transparency in North Ireland [7].

\section{RELATED WORK}

Blockchain technology can play a significant role in philanthropy to overcome many challenges. So, some studies have tried to utilize blockchain in a charity system. Firstly, Nor et al. [11] proposed Sadaqa system for disaster aid crowd funding. The system focuses on transferring funds to the people in need during the disaster using Ethereum smart contract to avoid problems of the modern day transactions related to transaction fees, potential fraud, absence of accountability, and transaction time.

Secondly, Hu et al. [12] proposed a charity system using Ethereum smart contracts, they leverage Blockchain to improve the transparency in charities and increase the public trust, the system allows donors to vote on the request of a beneficiary to involve donors in fundraising and enhance transparency.

Lastly, D. Jayasinghe et al. [13] built a Bitcoin payment system that can be used in both an offline environment via the existing GSM network, as well as, online environment to provide aid for people living in a challenging geographical environments with limited internet availability.

Based on the abovementioned studies, this article will investigate the crucial features in blockchain platforms to find the most suitable platform for charity system, and then it will propose a blockchain charity system that relies on a new model concentrates on enhancing transparency; the model contains elements that have not been addressed in the previous studies.

\section{BLOCKCHAIN}

Blockchain technology has gained widespread attention in recent years, as it promotes trust and transparency. In 2015 a report by the UK Charities Aid Foundation [14] points out that for charities, Blockchain helps to increase transparency, openness and confidence while reducing transaction costs and providing new opportunities for fundraising.

Blockchain can be identify as a public ledger, in which all transactions are stored in a chain of blocks; the ledger constantly grows when new blocks get approved and added by the network members [15].

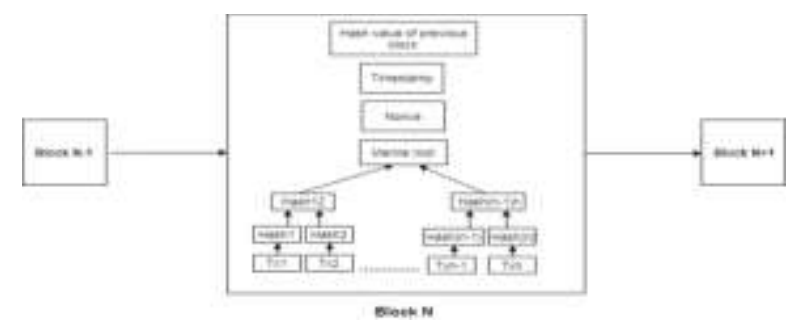

Fig. 2. Blockchain Structure.

Blockchain sorts and encrypts the information inside the block before adding the block to the chain. What makes the chain traceable is the blocks are linked with each other in the chain, giving each block encryption code called a hash [16]. Blockchain contains blocks where each block records transactions sent between users, hash code which is a unique code created when a new block is discovered, and a reference code of the previous block [17]. Fig. 2 illustrates how blocks are connected to form the Blockchain. Each block consists of transactions (Tx) that is represented in a hash number using Merkle tree, as well as other values such as hash value of previous block $(\mathrm{N}-1)$, timestamp, and nonce which is a random number that can be used just once, these values will be used to represent the hash value of the current block $(\mathrm{N})$, and this hash will be stored in the next block $(\mathrm{N}+1)$.

\section{KEY BENEFITS OF BLOCKCHAIN FOR BUSINESS}

Nguten and Hoang [18] pointed out the key benefits of blockchain technology that makes it attractive for many types of businesses, the benefits are:

1) Decentralized: blockchain networks are not controlled by a central party, which helps to avoid a single point of failure and take over the network by a small group of users. Users in Blockchain participate in a distributed network managed by consensus mechanism to reach the agreement on the network state.

2) Transparency: All network participants can see the stored data; in other words, the data stored in a blockchain is visible to the public.

3) Immutability: Once the data are stored in the Blockchain, it cannot be changed.

4) Security and privacy: One of the important features in the Blockchain is the cryptographically secure mechanism because it helps in promoting privacy and security. Furthermore, users in the network use a public and private key for identification and verification. When a transaction occurs, a user can be easily verified by his digital signature.

\section{Choosing a Suitable Blockchain Platform}

First of all, two crucial features have to be determined in the blockchain platform. The first step is to decide on the type of network, and then to decide whether Crypto-currencies is needed or not [19].

\section{A. Network Type}

Data cannot be exchange without networks; therefore, networking plays an important role in any field related to 
information technology. That is why there are vicarious network attacks such as mentioned by [20], [21], [22] , and [23].

With regard to the first step, there are three types of networks in blockchain: public network, private network, and consortium network.

1) Public network: It's a permission-less or open network, not controlled by any organization, anyone can join the network, and all users are equal; they can read and write without any restrictions. These blockchain networks are considered as fully decentralized. Transparency is the most significant advantage of the public network. However, there are some disadvantages of Public Network and that includes slow transaction speed as compared to other networks, and scalability issue though some steps are already taken to solve this problem such as off-chain, examples of public network Bitcoin and Ethereum [24].

2) Private network: The second type of the blockchain network is the private or permissioned network; it is centralized or partially-centralized as compared to the public network; it is controlled by a single organization that has the permission to write in the ledger, read can be public or restricted by participants. The advantages of this network are the transaction speed, and it is considered more scalable than a public network; however, it is not transparent since a single organization controls it. An example of a private network is Heyperledger, one of the popular private network platforms [25].

3) Consortium network: It is a semi-private network owned and controlled by a preselected group of members. This group of members has permission to write in the ledger, and read can be public or restricted by participants, it is more secure and has better scalability; however, it is less transparent. Marco Polo platform is an example of a consortium network [26].

Each blockchain network has unique features to offer; therefore, choosing a suitable platform would rely on the system requirements, for a charity system transparency has the highest priority. Thus, the public network is ideal for organizations that thrive on trust and transparency.

\section{B. Crypto-Currency}

The second feature is to decide on the need for cryptocurrencies. It is a currency that only exists digitally, that usually has no central issuing or regulating authority but instead uses a decentralized system to record transactions and handle the issuance of new units, and that relies on cryptography to avoid counterfeiting and fraudulent transactions.

The most important feature about crypto-currency is that it is not controlled by any central authority or a third party. It can be sent directly between two parties with minimum transaction fees and allow users to avoid the high fees that traditional institutions impose [13].
The crypto-currency was the first application of Blockchain presented by Nakamoto [27] in 2008 and it is known as Bitcoin. Since then, a lot of crypto-currencies have arisen, such as Ethereum, Tether, and many others.

There are many benefits that can be gained from Blockchain that would positively affect the existing charity systems. As pointed in previous studies [13] and [14], the benefits that can be obtained from using crypto-currency are:

1) Reducing transaction cost: One of the notable features of Blockchain is its low international transaction fees.

2) Transaction speed: In Ethereum, once a user clicks on send, the transaction will broadcast immediately, it takes some time to get confirmed, the time can be determined based on the gas amount that users are willing to pay.

3) Donation provisioning: Sending the donations to the recipient can be challenging in some cases. For instance, humanitarian financial aid distribution in war zones can be blocked if the country's bank system is subject to sanctions, Ethereum transactions can reach the recipient without needing to use any bank system.

\section{Smart Contract}

The third important feature is the smart contract. It is a computer program that allows the charity system to control the transferring of crypto-currencies and assets between users and these contracts are stored in a decentralized ledger. Many studies have suggested Smart Contract as a primary factor for charity system based blockchain [7], [10], and [13]. The first use of the smart contract was made by Ethereum [28].

It can also be defined as a set of instructions represented in computer code published on a distributed network that receives inputs, executes instructions, and provides outputs. It can enable a charity to over other features such as routine provisioning of donations, record keeping, donation requests to donors, and automatic audit reports of a charity activity [13].

\section{Consensus Algorithm}

The fourth important feature that should be taken into account is consensus algorithm or mechanism which is the backbone of the Blockchain and the core element of any blockchain platform. It plays a vital role to ensure the network's security, integrity, and performance [18], and [29].

The most popular consensus mechanism is Proof of Work (PoW) that was first introduced by Nakamoto [27]. It relies on the computing power to distribute a new block among network peers. PoW is used by many platforms such as Bitcoin and Ethereum. The second popular consensus is Proof of Stake (PoS) that was created as an alternative to PoW mechanism because of the high energy consumption of PoW consensus; PoS relies on the participant's stake rather than computing power. Different protocols have been introduced PoS approach with slight differences; such as Ouroboros [30] and Casper by Ehereum [31], another study suggested combining POW and POS such as Proof of Activity (POA) [24]. 
Furthermore, some platforms implement PoS, such as Peercoin, However, studies are still needed for more analysis and investigation for the Proof of Stake mechanism [29]. Thus, a specific algorithm will not be chosen, since it is currently under study, and it can be changed in the future. For example, Ethererum is planning to switch from PoW to PoS [31].

\section{DECISION SUPPORT SYSTEM}

Choosing a suitable platform for any type of business is an essential step before implementing blockchain [19]. However, there are many varieties of available blockchain platforms with multiple features. This problem called Multi-Criteria Decision-Making (MCDM), which is defined by "making preference decisions (such as evaluation, prioritization, and selection) over the available alternatives that are characterized by multiple, usually conflicting attributes" [32]. To cope with this problem, Farshidi et al.'s system [19] will be used.

Farshidi et al.'s system is called Decision Support System (DSS). The system formulates the Blockchain selected platform as an MCDM problem to find a suitable platform that supports the required features. They have extracted a set of blockchain features from online documentation of blockchain platforms. A list of the important features has been identified by researchers and experts.

Based on the DSS they are other features that need to be taken into account along with the abovementioned features to get the most suitable Blockchain platform, however, they are not crucial as the mentioned features, but they can help to differentiate between platforms. The features are:

1) Market positioning: It is measured by the Numerical feature like transaction speed, popularity in market and platform's maturity, and by Boolean feature, which is the number of innovations that platforms support, such as; Internet of Things and Artificial Intelligence, etc.

2) Capacity: It is measured by scalability technologies used, such as side-chains, Sharding, Plasma-chains, Off-chianstate-channels and On-Chain Transactions.

3) Development: It is measured by programming languages support, such as; Soildity, Python, JavaScripit, $\mathrm{C}++$, .Net and Golang.

4) Flexibility: It is measured by resilience technologies such as Sybil attack resistant, Spam-attack resistant, Quantumcomputing resistant, Instant transaction Finality, and Hardfork resistant.

5) Integration: Which means a platform that can integrate with the other systems, and it is measured by Interoperability technologies that supported by the platform such as Atomic Swap, Cross-chain technology, Enterprise system Integration.

The score will be indicated on a scale from 0 to 100 , where 0 represents the least suitable platform and 100 represents the most suitable platform. The following figure shows the result of the top 10 feasible platforms for the proposed system based on these features:
- Network type: public network.

- Smart contracts: yes.

- Crypto-currencies: yes.

- Consensus algorithms: Proof of Work or Proof of Stake.

- Market Positioning: High Transaction Speed, High Maturity, High Popularity, High Innovation.

- Programming languages support: Solidity, JavaScript and python.

- Interoperability technologies: any (Not specified).

- Scalability technologies: any (Not specified).

- Resilience technologies: any (Not specified).

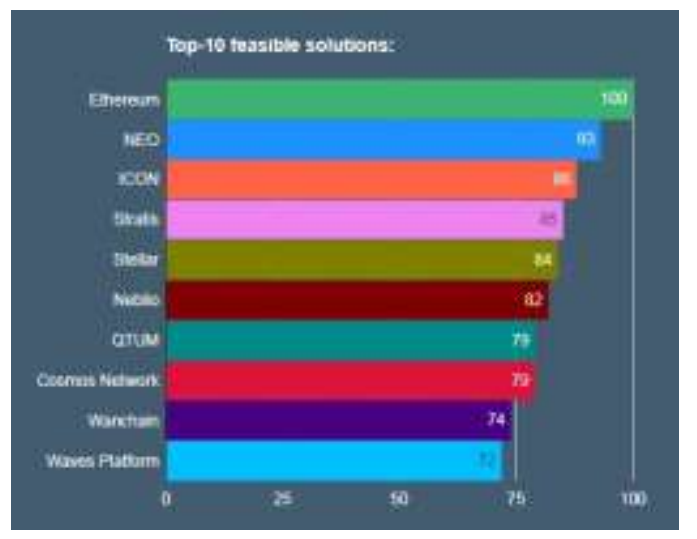

Fig. 3. The Result for the Suitable Platform based on Decision Support System [19].

Fig. 3 shows that Ethereum scored 100, which is the highest score among other platforms; because it supports all the selected features. NEO is the second platform scored 93; it has a lower score than Ethereum, because it does not support all features.

\section{ADDITIONAL ELEMENTS TO IMPROVE TRANSPARENCY}

As addressed above transparency issue in the current charitable organizations; and presented Blockchain as a solution that will help in tackling this issue. In addition to Blockchain other elements can be used, the following section discusses two important elements that can help in improving the transparency in charities.

\section{A. Monitoring Charitable Organization}

Evaluation and assessment of charitable organizations by another type of non-profit organization specialized in charity evaluation is one of the solutions to improve the transparency and give donors more confidence to contribute. This kind of organization called charity evaluator, and their job is ensuring that fundraising in charitable organizations is being organized and performed in a satisfactory manner and that the administration of the collected funds is adequate, one example on this organization is International Committee on Fundraising Organizations (ICFO) [33]. 
Therefore, having a Charity Evaluator organization in a charity will help in improving transparency and encouraging donors to donate confidently. It will also provide an additional layer of assurance in the prevention and detection of misuse of donors' funds.

\section{B. Offering a Refund Option}

Donazzan, Erkal, and Koh experiment showed that offering a refund option has a positive impact on giving behaviour and increasing confidence [34]. Another experimental study also showed that offering a refund option could increase the contributions [35]. Therefore, offering a refund option in a charity system has a potential to increase the confidence and the amount of contributions.

\section{SYSTEM MODEL}

Fig. 3 displays system processes flow; the system's main processes accrue through two main pages login page that lead to the user profile and view campaigns page to view campaign details.

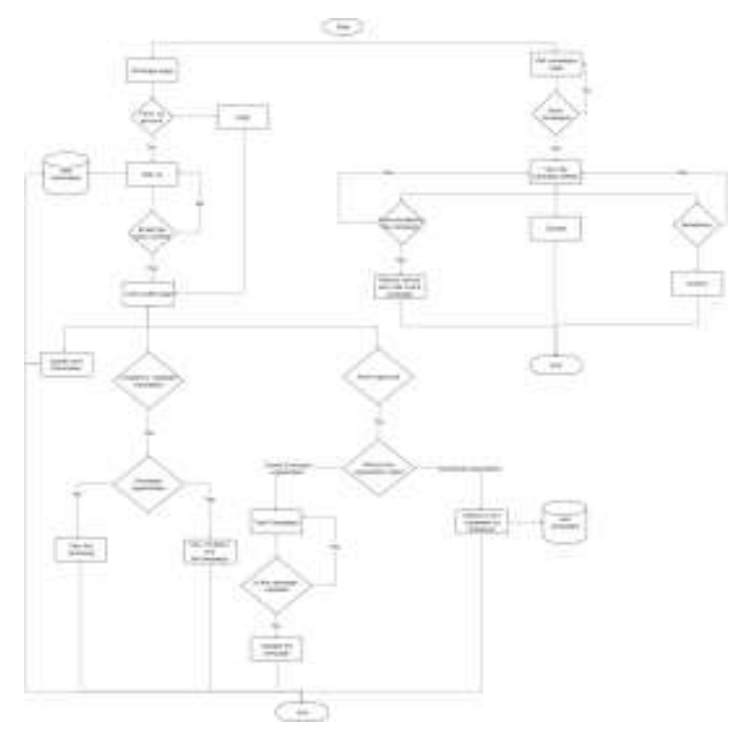

Fig. 4. System Flow Diagram.

According the Fig. 4, the system starts with two main processes, first login process for two types of user charitable organizations and evaluator organizations, they should login to interact with their campaign, to do so, they need the admin approval, if they received the approval, charitable organizations can create and deploy new campaigns on Ethereum network, and evaluator organizations can validate them.

On the other hand, campaigns will be accessible to the public without the need for logging in, so that donors, beneficiaries, and visitors can view campaigns' details that have been created by charitable organizations.

\section{The Design of THE System}

Fig. 5 illustrates the proposed system design; the system is a decentralized web application (Dapp), where the web application is (client side) that is connected to the Ethereum
Network (server side) through web3 that allows the system to interact with the Ethereum network. Users are required to use Metamask Ethereum wallet in order to interact with the system smart contracts, donors however, can donate by using Metamask or any other Ethereum wallet.

The Dapp will deploy the Campaign Factory smart contract once, so that the system users in particular the charitable organization can interact with this contract to deploy their campaigns. Additionally, the system will be able to track all campaigns that have been deployed through it.

Dapp users and visitors can view details of all campaigns contracts that have been deployed by charitable organizations. Donors can send money to the contract directly without the need to sign up, whereas, Charitable Organization and Charity Evaluator Organization should submit their information and wait till their application gets approved by Administrator. Once the Charitable organization's application is approved, they can interact and create any number of campaigns they want through a predefined Metamask wallet address. On the other hand, Charity Evaluator organizations are responsible for monitoring and evaluating charitable organizations' performance and campaigns.

Smart contract details with all transactions will be stored in the Ethereum blockchain network. Mysql database is used to store charitable organization and Charity Evaluator credentials and information.

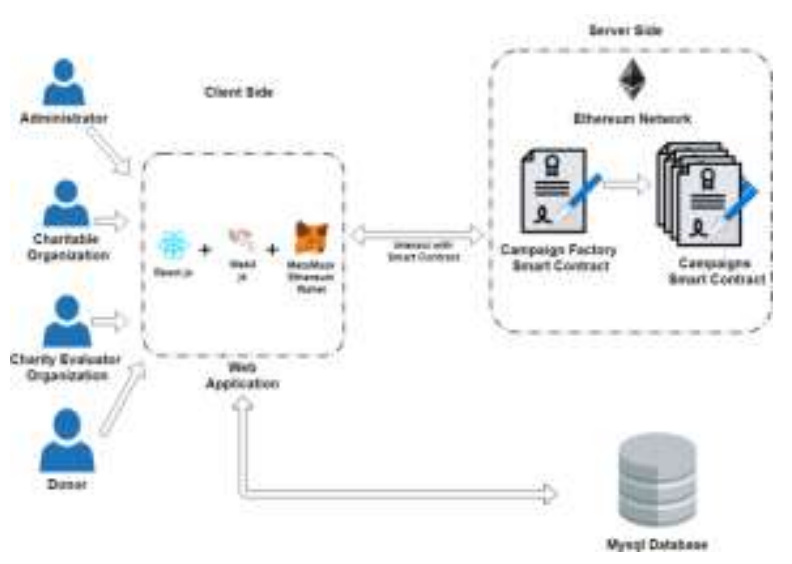

Fig. 5. Client Server System Architecture.

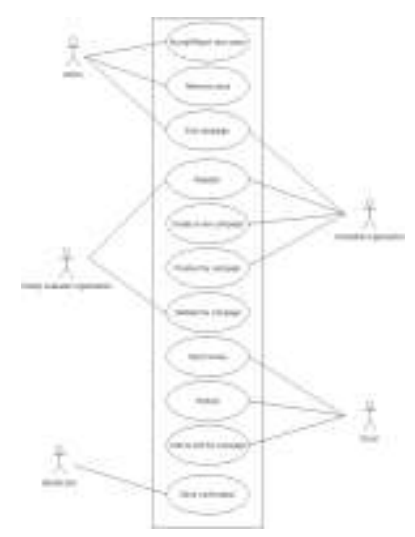

Fig. 6. Use Case Diagram. 
As shown in Fig. 6 they are four types of users in the proposed system:

1) Administrator: the Administrator is responsible for accepting or rejecting users' applications, as well as deleting users. He can also terminate any campaign's contract if he feels that the campaign does not meet the requirements.

2) Charitable organizations: this type of users has a profile; so, charitable organizations are required to provide information about the charitable organization's name, address, email, contact number, registration number, number of employees, mission and goals of the organization, in order to sign up to the Dapp. Then they need to verify their email address, upon which they will then be able to create new campaigns if their applications get approved by the Administrator. Charitable organizations' profile will be available and accessible to the public.

3) Charity evaluator organizations: they are similar to charitable organizations in terms of the signup process; however, their job is overseeing and monitoring the charitable organizations' campaigns and evaluating their performance.

4) Donors: they are not required to login in. Donors can send money through Metamask wallet or any Ethereum wallet to all campaigns' smart contract, and they also can get their money refunded any time during the lifetime of the campaign.

\section{SySTEM’s SMART CONTRACTS}

The proposed system has two smart contacts.

\section{A. Campaign Factory Smart Contract}

Campaign Factory is a smart contract that has been deployed before the launch of the Decentralized Application (Dapp). This is to enable the charitable organization to deploy their smart contract (Campaigns) through Campaign Factory. Therefore, Dapp will be able to track all campaigns that have been deployed by the charitable organizations. This contract has two functions:

- To create a campaign: this function invokes by a charitable organization to create a new contract, the function's parameters are minimum target amount of Ether needed to finalized the campaign, originator Metamask wallet address, description about the campaign, campaigns parties' information, beneficiary wallet address, and the end date of the campaign.

- To list deployed campaigns: the Dapp calls this function to display a list of all campaigns that have been deployed by charitable organizations.

\section{B. Campaign Smart Contract}

A charitable organization deploys this contract through the Campaign Factory smart contract; parties involved in this contract are Charitable Organization, Charity Evaluator, Donors and Beneficiary. Campaign smart contract shows information about the campaign and receives Ether from donors, as well as, holds donors Ether until all conditions stated in the contract are fulfilled. Later, it will allow a charitable organization to finalize its campaign; once the campaign gets finalized, the funds will be sent to the beneficiary. Once the campaign is completed, no one can interact with the finalized contract, and it will remain as a reference that stored all transaction and information. The Campaign contract performs the following functions:

- Donate(): this function allows donors to donate for the campaign through the website application by using Meta mask Ethereum Wallet extension in the browser. When a user clicks on a donate button, the website will invoke the donate function from the Campaign smart contract, and Ether will be sent to the contract.

- Receive(): this function gets called when the donors send money from any Ethereum wallet other than Metamask wallet. It allows the Campaign smart contract to receive Ether from anywhere.

- Refund(): this function gives donors the right to get their money refunded at any time they want as long as the contract has not been finalized.

- CharityEvaluatorApproval(): Any charity evaluator organization can execute this function, it will change the value of ApprovedByEvaluator from false to true, and this will indicate that a charity evaluator organization has approved the campaign.

- BeneficiaryApproval(): Similar to the Charity Evaluator Approval function, beneficiary confirmation is also required; beneficiary only can call this function.

- Finalize(): This function can be called only by contract originator (the charitable organization) in order to transfer the money to the beneficiary.

- TerminatetheCampaign(): The contract originator or the Administrator can call this function. It sends the Ether back to donors and blocks any future interactions with this campaign.

- VotetoTerminate(): The function can be called by a donor who has donated to the campaign to vote for termination of the campaign.

- GetSummary(): This function gets called by the Dapp to display campaign details to the public.

Note that Metamask Ethereum wallet is required for all parties if they want to interact with the deployed contract, except, contribution or sending money to the campaign can be either by Metamask or other Ethereum wallets. Campaign smart contract rules are:

- Once the contract gets deployed, funds will be held in the smart contract in the Ethereum blockchain network till the campaign ends.

- When the campaign ends, only a charitable organization can finalize the contract to transfer the funds to the beneficiary address.

- A charitable organization cannot finalize the campaign without other parties' confirmation (Charity Evaluator and Beneficiary), should have passed campaign end 
date, and the funds should reach the minimum amount of Ether.

- During the lifetime of the campaign contract, donors can get their Ether refunded at anytime they want.

- During the lifetime of the campaign donors can vote to terminate the campaign if they are not satisfied. If end date has already passed and $50 \%$ of the donors are not satisfied with the campaign the charitable organization (campaign originator) will not be able to finalize the contract, and they will be required to terminate the campaign.

- If the campaign end date has already passed, and the contract's conditions are not fulfilled, a charitable organization or Administrator can refund Donors money by calling the termination function.

- Once funds are sent successfully to the beneficiary, the campaign will not accept any new transaction, and no one can interact with it. However, all transactions and information will remain available and accessible to the public.

- Charitable organization (campaign originator) and Administrator can terminate the campaign at any time, when they do so, consequently, funds will be sent back to donors and the campaign will not accept any other transactions.

\section{Multi-Point MOdEL}

Fig. 7 illustrates how the Multi-point approach has been applied in the campaign smart contract; the campaign should go through seven processes, each of which is designed for a certain player.

For example when a charitable organization wants to finalize its campaign, the request through MetaMask wallet will be sent to the campaign smart contract in a transaction form to invoke the 'finalize function'. The campaign contract will first check if the sender ID is same as the contract originator ID to ensure that only the campaign originator (charitable organization) can finalize the campaign. It will then check if the campaign is validated by a charity evaluator organization, and verify that the beneficiary has confirmed the campaign and that $50 \%$ or more of donors are satisfied with the campaign. The campaign contract will then check if the campaign target is achieved and the campaign has reached its end date. Only when these conditions are met, will the transaction be confirmed and the campaign is finalized.

As shown in Fig. 6 the first step is to check the campaign status. If the campaign is finalized by its originator, users will no longer be able to interact with the finalized campaign. They can however continue to read the campaign details. The important point of this approach is to ensure that before the campaign is finalized or completed, it has to go through Multipoint campaign finalization process. This process would involve various parties in the fundraising process.

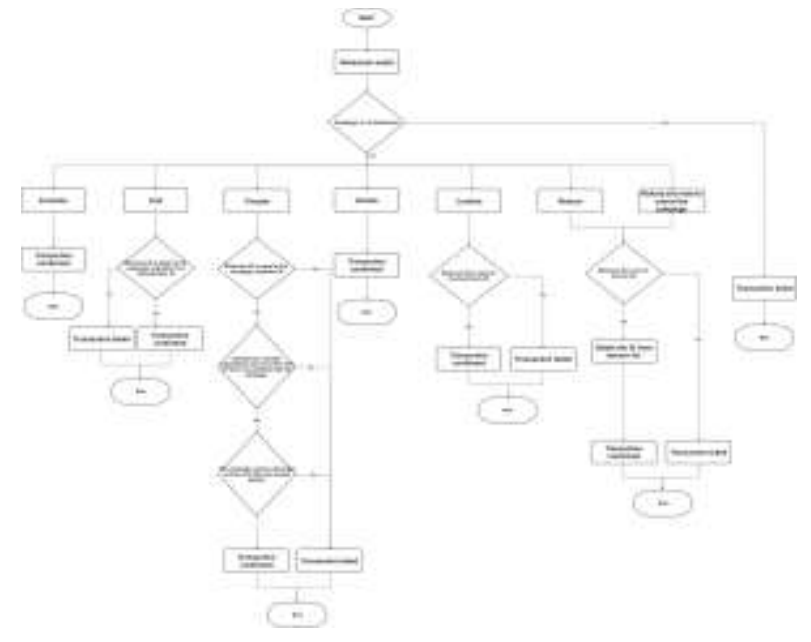

Fig. 7. Multi-point Model.

\section{SYSTEM INTERFACE}

The system pages will be shown in this section, the system is a Dapp. The following points and figures illustrate the system pages, the Dapp interface consists of:

- Home page layout: Fig. 8 shows the home page of the Dapp. It is the first page that will be displayed for users - the first page that users will view in the website.

This page provides a short description about Blockchain and Smart Contract, as well as the latest two campaigns run by charitable organizations are displayed. From the navigation menu, user can go to the campaigns, about us, login and sign up pages.

- Campaigns page layout: as shown in Fig. 9 on this page users can check all campaigns that have been created by charitable organizations through the Campaign Factory smart contract. A list of campaigns will be displayed, consisting its campaign title, charitable name, short description about the campaign, and campaign status (to show if the campaign is completed).

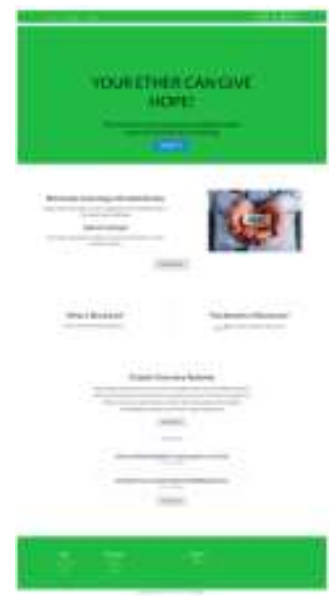

Fig. 8. Home Page Layout. 


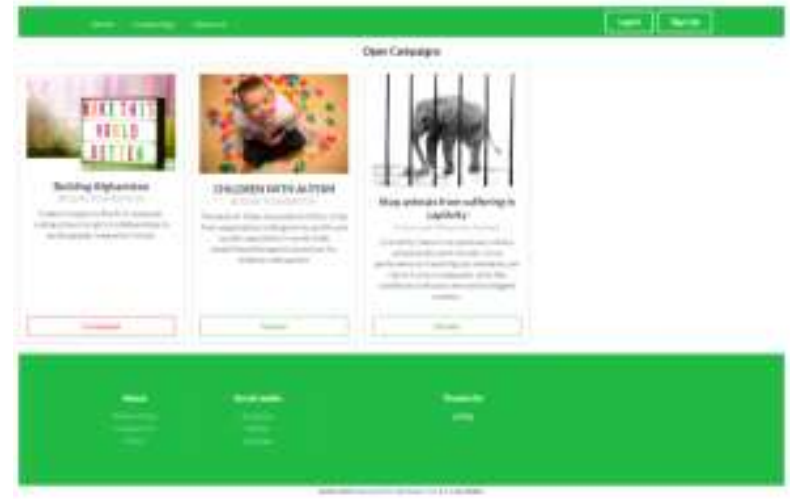

Fig. 9. Campaigns Page Layout.

- About us page: This page includes system directors' personal information such as name and email address. This page is also accessible to the website visitors to enable them to get in touch with the board members should the needs arise. Fig. 10 shows this page layout.

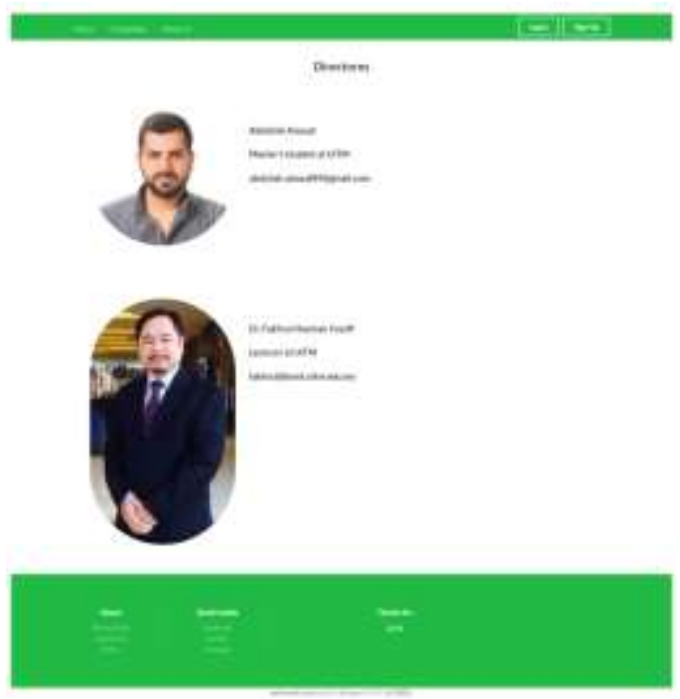

Fig. 10. About us Page Layout.

- Signup page layout: Fig. 11 illustrates this page layout. On this page the two types of users allowed to sign up are the charitable organization and charity evaluator organization. Users are required to fill fields that are divided into four main sections; first section is login information which includes user email and password. The second section includes organization name, mission and goals (for charitable organization) and standards (for charity evaluator organization), number of employees, address, and the registration number of the organization. The third section is the contact information such as phone number, email, and website. In the last section users are required to enter theirs Ethereum wallet address.

- Login page: as shown in Fig. 12 from this page users can access their accounts once their credentials are keyed in. The two fields that users are required to fill in are their email and password. If a user doesn't have an account yet, he/she should go to the sign up page through a hyperlink provided underneath the fields form, or through the menu bar to create a new account.

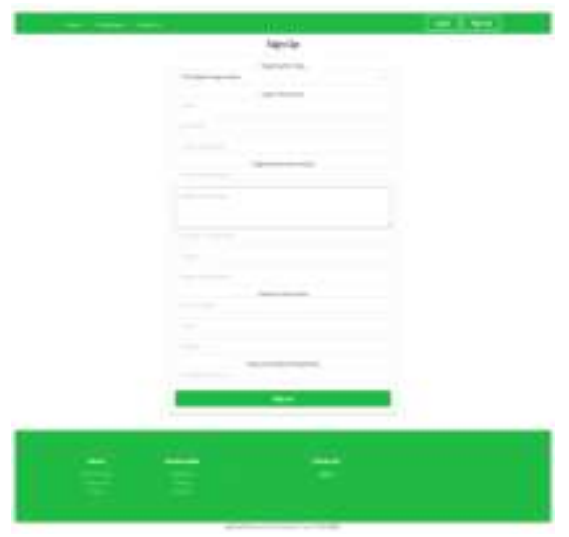

Fig. 11. Signup Page Layout.

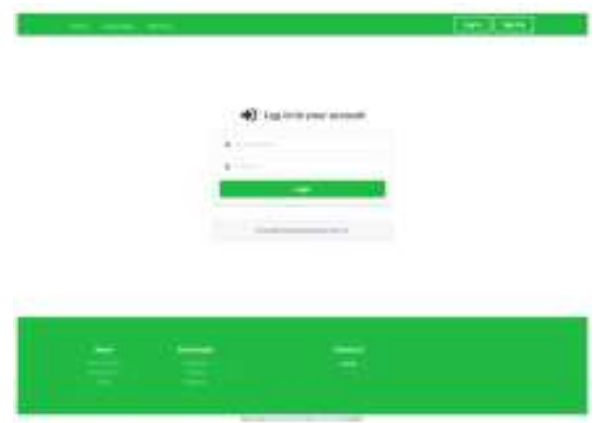

Fig. 12. Login Page Layout.

- Campaign details page: as Fig. 13 shows, this page displays all the details of a campaign; it is the main page of the Dapp since most of the interactions with the Campaign smart contract will take place on this page. Here, donors can donate to a campaign. The second section is used to show information about the originator of the campaign (charitable organization), which includes name and Ethereum address of the organization as well as a button to visit the organization's profile and a hyperlink to send users to the Etherscan to check its account. Details on the charity evaluator information are also displayed here. 'Waiting for validation from evaluator' message will be prompted if the campaign has yet to be validated by a charity evaluator. The beneficiary information is shown at the bottom of the page: as who is/are the beneficiary/ies, their physical address along with their Ethereum address and a hyperlink to visit their account in the Ethereum network. Underneath the beneficiary information section, unhappy donors could claim for a refund and vote to cancel the campaign. In addition, campaign information is shown under the campaign image - campaign title, short description about the campaign, and campaign status (it will be funded if the campaign is still running and completed if the campaign is completed and does not accept a new transaction). The minimum amount of funding needed for this the campaign is displayed with a progress bar 
to show the percentage of the campaign balance, and donors rating (if a donor had requested for a refund of his Ether and voted to cancel the campaign hence affecting the rating). The same page also displays the campaign details such as start and end date, campaign target (the minimum amount), campaign balance, number of donors, number of unsatisfied donors, campaign manager name and email, campaign Ethereum address, and campaign goals.

Unlike the general users, the two types of logged in users (charitable organization and evaluator organization) can also see/access to the following buttons:

1) Evaluator organization's button: This button is for evaluator organizations to validate a campaign.

2) Charitable organization's button: Only charitable organizations can see this button to either finalize or terminate the campaign.

- Charitable organization's profile: After a user has verified his account and logged in, he needs to wait until his account gets approved by the admin. If approved, new campaign button will be appeared on the user's page as it is shown in Fig. 14 and he will then be able to update certain information such as address, number of employees, phone number, contact email, missions and goals, and email. The user will also be able to see his campaigns and access it to finalize, terminate, or just check on the campaign status.

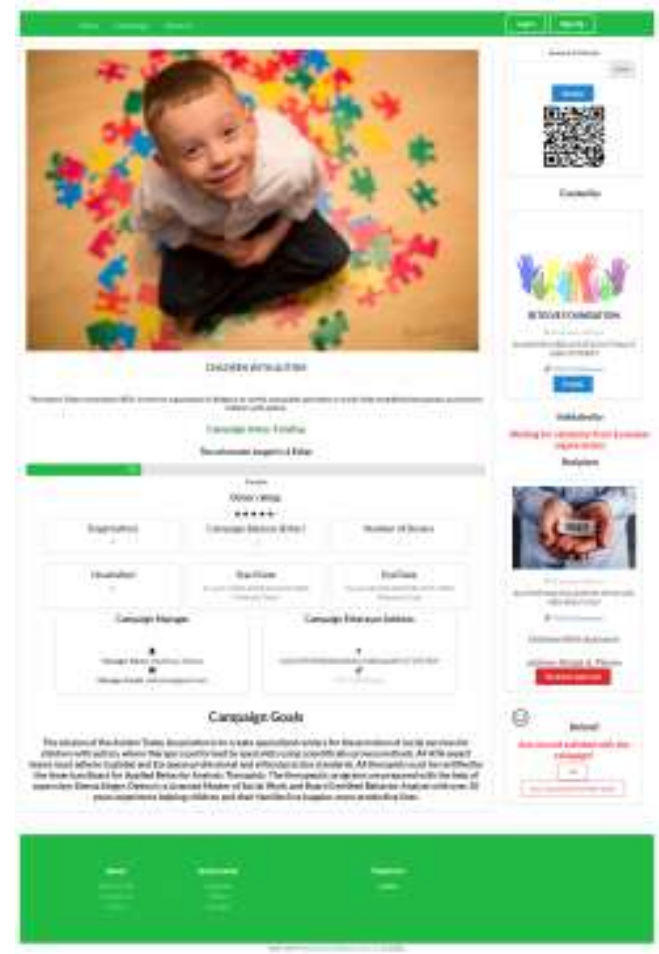

Fig. 13. Campaign Details Page Layout.

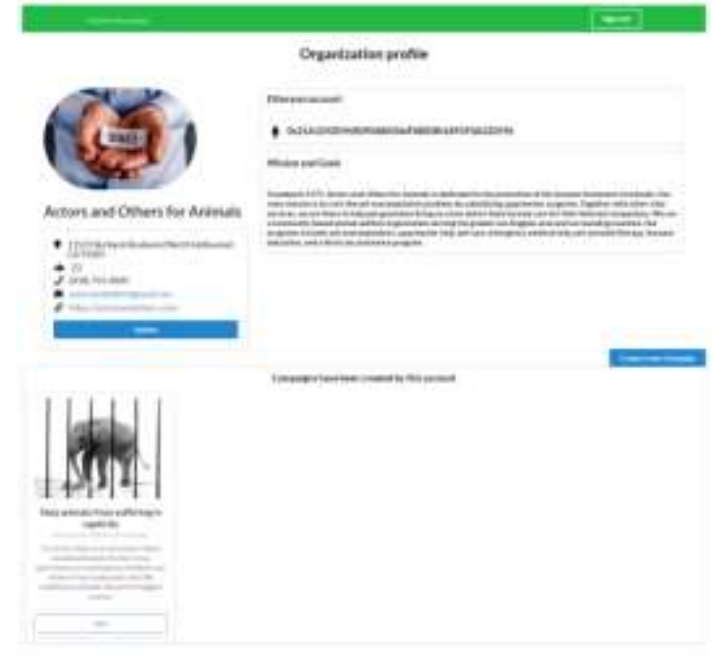

Fig. 14. Charitable Organization's Profile Layout.

As shown in Fig. 15, if the user wants to create a new campaign, a new page will be opened. This page is only accessible to the charitable organizations. To create a new campaign, the user will furnish some relevant information on the campaign, i.e. title, short description about the campaign, minimum target needed to fulfil campaign goals, beneficiary Etherum address so that the funds will be automatically transferred to this account after the campaign is completed and finalized, campaign goals, beneficiary information, campaign manager information, and end date.

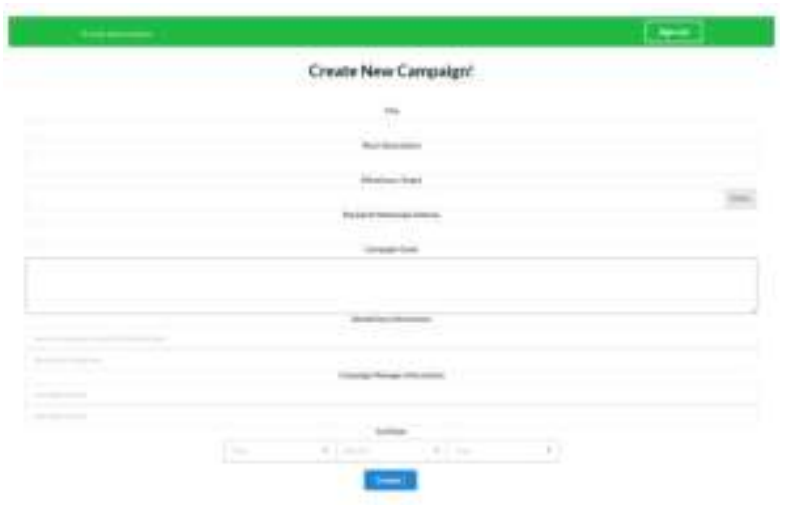

Fig. 15. Create New Campaign Page Layout.

The user must use his predefined Metamask address so that after clicking on the create button, his MetaMask wallet will pop up and display the transaction fee. Once the user clicks on the confirm button, only then will his new campaign be deployed in the Ethereum network.

- Charity Evaluator Organization: as Fig. 16 shows the charity evaluator organizations' profile is very similar to the charitable organization. After a user verifies his account and logged in, he needs to wait until he gets the admin's approval. 


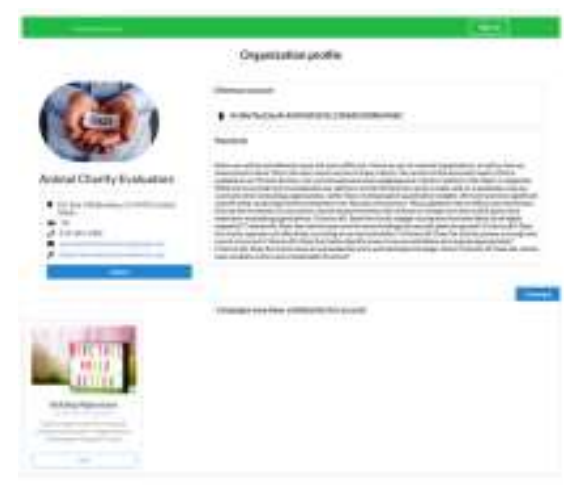

Fig. 16. Charity Evaluator's Profile Layout.

Similarly, when the user account gets approved, a campaign button will be appeared in his account and the user will be able to update certain information such as address, number of employees, phone number, contact email, standards, and email address.

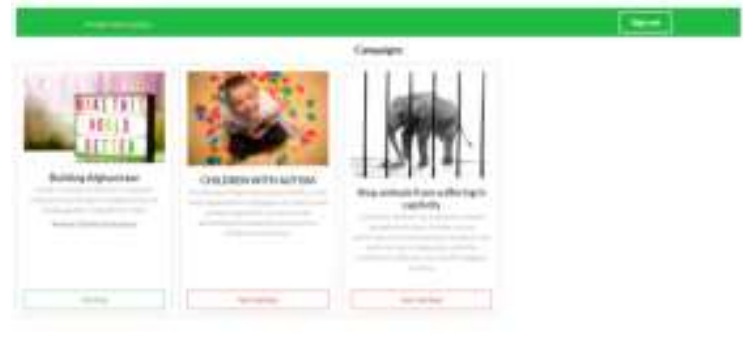

Fig. 17. Charity Evaluator Campaigns Page Layout.

The user can also see those campaigns that have been validated by him as shown in Fig. 17. When the evaluator clicks on the campaigns buttons on the page, a list of campaigns will be displayed and the user can then access to information such as the campaign details and whether or not the campaigns are validated.

\section{TEST SySTEM's SMART CONTRACT}

The Dapp contracts have been tested locally; Rinkeby Ethereum test network is used during the test to obtain virtual Ethereum tokens for donation and expenses required for the test.

Infura provides a suitable entry point for the Rinkeby network. MetaMask wallet was used to store the Ethereum tokens and complete the corresponding transactions. At the same time, a Web3 instance completes the interaction between the website and the Ethereum network. Table I shows accounts and their balances that have been utilized for testing before the campaign is being deployed.

The campaign includes a description of the campaign, goals, information about the Originator and the Beneficiary, end date of the campaign to notify the public that the Originator will be able to finalize the campaign after that date, it also displays the campaign balance along with all transactions that have been made and the minimum target amount needed to achieve campaign's goals (the minimum target for this campaign is 1 Ether).

Table II illustrates all interactions with System contracts; the first transaction made by Originator to create a new campaign. $0.00141 \mathrm{ETH}$ of the gas limit was used to execute the create function in the campaign factory smart contract. The second transaction was made by the Donor A to send 0.25 Ether from his wallet to the testing campaign smart contract, the gas fee used to execute this transaction was 0.00012 Ether. Donor B also donated with Ether to the campaign at a different amount of 0.10 Ether. After that, the Originator tried to finalize the campaign, but the transaction failed because some requirements have not yet been fulfilled.

TABLE I. ETHEREUM ACCOUNTS THAT HAVE BEEN USED FOR TESTING THE SMART CONTRACT

\begin{tabular}{|l|l|l|}
\hline Account & Address & Balance $($ ETH $)$ \\
\hline Originator & $\begin{array}{l}\text { 0x38e7be22eaFc465042f1b92c13D68 } \\
\text { 5342Bb034AC }\end{array}$ & 1 \\
\hline Monitor & $\begin{array}{l}\text { 0xbB1E68cE914f95b4aB86d81581Bb } \\
\text { 25aB5C2B3402 }\end{array}$ & 1 \\
\hline Beneficiary & $\begin{array}{l}\text { 0x4a6F69e31BE1a5A3E4d7A794ba7 } \\
\text { 6a2Ba71f9DB93 }\end{array}$ & 0.0007 \\
\hline Donor A & $\begin{array}{l}\text { 0x21A1292D940090AB830eF88D0fb } \\
\text { 1891F0A220596 }\end{array}$ & 1 \\
\hline Donor B & $\begin{array}{l}\text { 0x0C69Ff3624c607Bc3Aca6BF8f2D } \\
\text { 5f93bb3B4bDD3 }\end{array}$ & 1 \\
\hline Donor C & $\begin{array}{l}\text { 0x2700556Ab9a1eb2D39c1D53C22b } \\
\text { 40E5982671457 }\end{array}$ & 1 \\
\hline
\end{tabular}

TABLE II. ALL INTERACTIONS WITH THE CAMPAIGN SMART CONTRACT

\begin{tabular}{|c|c|c|c|}
\hline Originator & Action & Message & Notes \\
\hline Manager & $\begin{array}{l}\text { Create a new } \\
\text { campaign }\end{array}$ & $\begin{array}{l}\text { Confirmed } \\
\text { Transaction }\end{array}$ & - \\
\hline Donor A & $\begin{array}{l}\text { Donate }(0.25 \\
\text { Ether })\end{array}$ & $\begin{array}{l}\text { Confirmed } \\
\text { Transaction }\end{array}$ & Contract balance 0.25 Ether \\
\hline Donor B & $\begin{array}{l}\text { Donate }(0.10 \\
\text { Ether) }\end{array}$ & $\begin{array}{l}\text { Confirmed } \\
\text { Transaction }\end{array}$ & Contract balance 35.0 Ether \\
\hline Originator & $\begin{array}{l}\text { Finalize the } \\
\text { campaign }\end{array}$ & $\begin{array}{l}\text { Failed } \\
\text { Transaction }\end{array}$ & $\begin{array}{l}\text { Failed transaction: because the } \\
\text { contract has not been } \\
\text { confirmed and verified by the } \\
\text { beneficiary and the monitor. }\end{array}$ \\
\hline Beneficiary & Approval & $\begin{array}{l}\text { Confirmed } \\
\text { Transaction } \\
\end{array}$ & - \\
\hline Originator & Approval & $\begin{array}{l}\text { Confirmed } \\
\text { Transaction } \\
\end{array}$ & - \\
\hline Manager & Finalize & $\begin{array}{l}\text { Failed } \\
\text { Transaction }\end{array}$ & $\begin{array}{l}\text { Failed transaction: the end } \\
\text { date has not passed yet, and } \\
\text { the target balance has not } \\
\text { reached. }\end{array}$ \\
\hline Donor C & $\begin{array}{l}\text { Donate }(0.65 \\
\text { Ether) }\end{array}$ & $\begin{array}{l}\text { Confirmed } \\
\text { Transaction }\end{array}$ & Contract balance is 1 Ether \\
\hline Originator & Finalize & $\begin{array}{l}\text { Failed } \\
\text { Transaction }\end{array}$ & $\begin{array}{l}\text { Failed Transaction: because } \\
\text { the end date has not passed } \\
\text { yet. }\end{array}$ \\
\hline Originator & Finalize & $\begin{array}{l}\text { Confirmed } \\
\text { Transaction }\end{array}$ & $\begin{array}{l}\text { The contract finalized and the } \\
\text { Ether sent to the beneficiary, } \\
\text { no more interaction can be } \\
\text { done. }\end{array}$ \\
\hline
\end{tabular}


The campaign includes a description of the campaign, goals, information about the Originator and the Beneficiary, end date of the campaign to notify the public that the Originator will be able to finalize the campaign after that date, it also displays the campaign balance along with all transactions that have been made and the minimum target amount needed to achieve campaign's goals (the minimum target for this campaign is 1 Ether).

Finally, the originator was able to finalize the contract and transfer the money to the Beneficiary when all the requirements and conditions have been fulfilled.

Table III presents the accounts' balance after the Testing Campaign has been finalized. All of the Testing Campaign's transactions with time and fees are recorded on Ethereum blockchain as shown in Fig. 18. The figure is taken from Etherscan website, a search engine that lets users look up, confirm and validate transactions on the Ethereum network.

TABLE III. ETHEREUM ACCOUNT AFTER THE CAMPAIGN SMART CONTRACT IS FINALIZED

\begin{tabular}{|l|l|l|}
\hline Account & Address & Balance $($ ETH $)$ \\
\hline Originator & $\begin{array}{l}\text { 0x38e7be22eaFc465042f1b92c13D6853 } \\
\text { 42Bb034AC }\end{array}$ & 0.998501 \\
\hline Monitor & $\begin{array}{l}\text { 0xbB1E68cE914f95b4aB86d81581Bb2 } \\
\text { 5aB5C2B3402 }\end{array}$ & 0.99997 \\
\hline Beneficiary & $\begin{array}{l}\text { 0x4a6F69e31BE1a5A3E4d7A794ba76a } \\
\text { 2Ba71f9DB93 }\end{array}$ & 1.000616 \\
\hline Donor A & $\begin{array}{l}\text { 0x21A1292D940090AB830eF88D0fb1 } \\
\text { 891F0A220596 }\end{array}$ & 0.749872 \\
\hline Donor B & $\begin{array}{l}\text { 0x0C69Ff3624c607Bc3Aca6BF8f2D5f9 } \\
\text { 3bb3B4bDD3 }\end{array}$ & 0.89988 \\
\hline Donor C & $\begin{array}{l}\text { 0x2700556Ab9a1eb2D39c1D53C22b40 } \\
\text { E5982671457 }\end{array}$ & 0.349902 \\
\hline
\end{tabular}

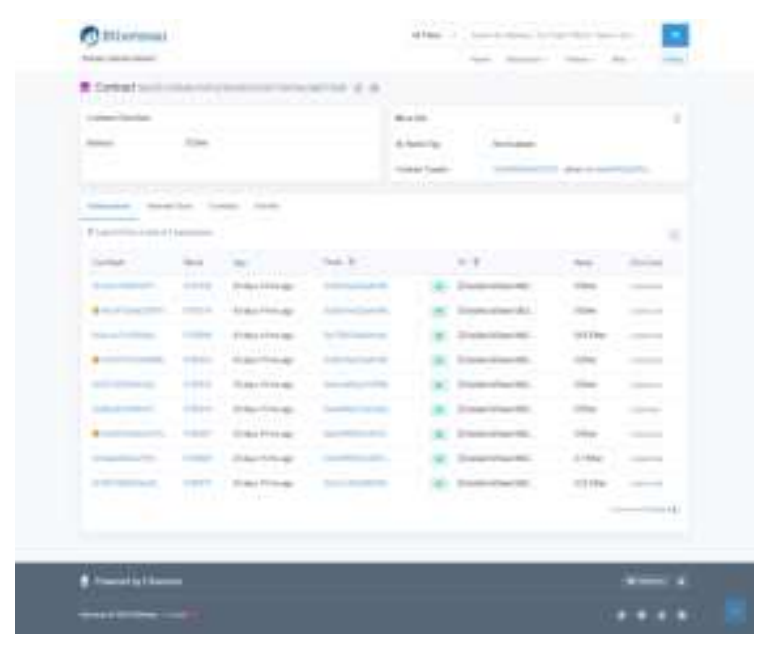

Fig. 18. Etherscan All Transaction Details.

\section{Discussion AND Results}

The study proposes the Multi-point approach to tackle transparency issue, in which the fundraising process should go through multi acceptance and specific conditions before it gets finalized. In other words, rather than having a campaign managed and controlled by one party, this approach involves other parties between the charitable organization and the beneficiary and empower them to improve the transparency in fundraising. Thus, that could help in strengthening the relationship between the charitable organization and donors. Furthermore, it will create a transparent environment that could affect positively on the charitable organizations and increase the quantity and amount of donations to charities, as well as boost donors trust.

The biggest issue in the existing Dapps' campaigns is that they are fully controlled by one party which is the campaign's originator, so they can spend funds anytime and anywhere without any constraints. The proposed Dapp allows donors to play a significant role in the campaign. When the contract gets finalized by the originator the funds will be sent automatically to one direction (beneficiary address) that has already been defined in the campaign.

In addition, existing Dapps do not offer refund option nor do they ensure that the donated funds are sent to the beneficiary. On the other hand, the proposed Dapp ensures that donors can get their money back at any time during the lifetime of the campaign.

The Multipoint approach in fundraising is concerned about empowering donors and involving charity evaluator organizations in the fundraising, between a charitable organization and a beneficiary, in which, charity evaluator organizations are responsible for evaluating and monitoring charitable organizations' campaigns. With the presence of the charity organization evaluators who act as a party ensuring transparency and authenticity of a charity body, donors involved in the fundraising process are protected from fraudulent charities. Moreover, donors can evaluate campaigns and play a major role in the fundraising process. The Dapp interacts with two smart contracts: the Campaign Factory smart contract that uses for creating and tracking charities' campaigns, and the Campaign smart contract that creates by a charitable organization to receive and hold donors funds. Based on certain conditions such as the campaign status, charity evaluator's evaluation, and donor satisfaction, the smart contract determines whether or not the funds can be sent. Besides, all interactions (transactions) with the Campaign smart contract are accessible and traceable and they will be recorded in the Ethereum blockchain.

Finally, based on the above discussion the Dapps's smart contract has been tested and has proven that it can deal with the lack of transparency issue in charity, in consequence, rebuild trust and confidence in charity.

\section{XV.CONCLUSION}

Many people have a passion for contributing to society, and they want to donate generously to charitable organizations. However, the lack of transparency in charity caused a trust issue. Transparency is essential in fundraising to maintain public trust, and should be the top priority for charity organizations. Therefore, the research proposed a Multi-point 
approach based on the usage of Blockchain technology to overcome the transparency issue in charity. First, it investigated the potentials of Blockchain can improve transparency, and then it analyzed the important features related to Blockchain that should exist in the charity's Blockchain platform. The significant features are public network, crypto-currency, smart contract, and consensus algorithm. Finally, the system was tested and proved that it can enhance transparency.

\section{ACKNOWLEDGMENT}

Special thanks to Faculty of Computer and Mathematical Science (FSKM) at Unversiti Technolgi MARA, Shah Alam for all the reference resources and support.

\section{REFERENCES}

[1] O. Dijk and M. Holmén, "Journal of Behavioral and Experimental Economics Charity , incentives , and performance R," vol. 66, pp. 119128, 2017.

[2] E. Ahmad, "Pakistan Institute of Development Economics Food Insecurity in Pakistan :," no. September, 2018.

[3] N. Hyndman and D. Mcconville, "Trust and accountability in UK charities : Exploring the virtuous circle," Br. Account. Rev., vol. 50, no. 2, pp. 227-237, 2018.

[4] J. T. A. Prakash, "Will You Trust Me?: How Individual American Donors Respond to Informational Signals Regarding Local," Volunt. Int. J. Volunt. Nonprofit Organ., vol. 28, no. 2, pp. 621-647, 2017.

[5] D. Mcdonnell and A. C. Rutherford, "Promoting charity accountability: Understanding disclosure of serious incidents it? , Account. Forum, no. September 2017, pp. 1-11, 2018.

[6] A. Hind, "New development: Fundraising in UK charities-stepping back from the abyss," Public Money Manag., vol. 37, no. 3, pp. 205210, 2017.

[7] I. Mori, C. Commission, and N. Ireland, "Public trust and confidence in charities," 2016.

[8] A. MacHado, M. Sousa, and Á. Rocha, "Blockchain Technology in Education," ACM Int. Conf. Proceeding Ser., vol. 12, no. 5, pp. 130134, 2020.

[9] H. B. M. Hadzir and F. H. Bin Yusoff, "Blockchain Based Data Structure for Travel Entourage Tracking System," 2019 4th Int. Conf. Inf. Syst. Comput. Networks, ISCON 2019, pp. 538-541, Nov. 2019.

[10] H. F. Atlam and G. B. Wills, Technical aspects of blockchain and IoT, 1st ed. Elsevier Inc., 2018.

[11] R. M. Nor, M. M. H. Rahman, and T. Rahman, "Blockchain Sadaqa Mechanism for Disaster Aid Crowd Funding," no. 211, pp. 400-405, 2017.

[12] B. Hu and H. Li, "Research on Charity System Based on Blockchain," IOP Conf. Ser. Mater. Sci. Eng., vol. 768, no. 7, 2020.

[13] D. Jayasinghe, S. Cobourne, and K. Markantonakis, "Philanthropy On The Blockchain," 2012.

[14] Charities Aid Foundation, "Giving Unchained: Philanthropy and the Blockchain," Charities Aid Foundation, 2015. [Online]. Available: https://www.cafonline.org/about-us/publications/2015-publications/ giving-unchained-philanthropy-and-the-blockchain.

[15] X. Chen, "Blockchain challenges and opportunities: a survey Zibin Zheng and Shaoan Xie Hong-Ning Dai Huaimin Wang," vol. 14, no. 4, pp. $352-375,2018$.

[16] M. A. Jamison and P. Tariq, "Five things regulators should know about blockchain ( and three myths to forget )," Electr. J., vol. 31, no. 9, pp. 20-23, 2018.

[17] J. Herbert and A. Litchfield, "A Novel Method for Decentralised Peer to - Peer Software License Validation Using Cryptocurrency Blockchain T echnology," no. January, pp. 27-30, 2015.

[18] C. T. Nguyen and D. T. Hoang, "Proof-of-Stake Consensus Mechanisms for Future Blockchain Networks: Fundamentals, Applications and Opportunities," vol. 7, pp. 85727-85745, 2019.

[19] S. Farshidi, S. Jansen, S. Espana, and J. Verkleij, "Decision Support for Blockchain Platform Selection: Three Industry Case Studies," IEEE Trans. Eng. Manag., vol. PP, pp. 1-20, 2020.

[20] N. S. Selamat, F. H. M. Ali, and N. A. A. Othman, "Polymorphic malware detection," 2016 6th Int. Conf. IT Converg. Secur. ICITCS 2016, Nov. 2016.

[21] M. A. M. Yusof, F. H. M. Ali, and M. Y. Darus, "Detection and Defense Algorithms of Different Types of DDoS Attacks Using Machine Learning," Lect. Notes Electr. Eng., vol. 488, pp. 370-379, 2018.

[22] S. M. Jawi and F. H. M. Ali, "Rules and results for SSL/TLS nonintrusive proxy based on JSON data," 2016 6th Int. Conf. IT Converg. Secur. ICITCS 2016, Nov. 2016.

[23] M. Azizi and M. Ariffin, "Data Leakage Detection in Cloud Computing Platform Investigation and Review of Cloud Computing Security View project," Artic. Int. J. Adv. Trends Comput. Sci. Eng., vol. 8, no. 1, pp. 400-408, 2019.

[24] I. Bentov, C. Lee, and A. Mizrahi, "Proof of Activity: Extending Bitcoin 's Proof of Work via Proof of Stake," no. 240258, pp. 1-19, 2013.

[25] W. Viriyasitavat and D. Hoonsopon, "Journal of Industrial Information Integration Blockchain characteristics and consensus in modern business processes,” J. Ind. Inf. Integr., vol. 13, no. June 2018, pp. 32-39, 2019.

[26] A. Banerjee, Blockchain with IOT: Applications and use cases for a new paradigm of supply chain driving efficiency and cost, 1st ed., vol. 115 . Elsevier Inc., 2019.

[27] S. Nakamoto, "Bitcoin : A Peer-to-Peer Electronic Cash System," 2008.

[28] Ethereum, "Ethereum Whitepaper | Ethereum.org," 2013. [Online]. Available: https://ethereum.org/whitepaper/. [Accessed: 11-Jun-2020].

[29] E. Deirmentzoglou and G. Papakyriakopoulos, "A Survey on LongRange Attacks for Proof of Stake Protocols," IEEE Access, vol. 7, pp. 28712-28725, 2019.

[30] A. Kiayias, A. Russell, B. David, and R. Oliynykov, "Ouroboros : A Provably Secure Proof-of-Stake Blockchain Protocol,” 2019.

[31] V. Buterin and V. Griffith, "Casper the Friendly Finality Gadget," pp. 1$10,2019$.

[32] C. L. Hwang and K. Yoon, "Multi-objective decision making-methods and application. A state-of-the-art study," New York Springer-Verlag, 1981.

[33] W. Burkhard, "Monitoring Charitable Organizations: Criteria and Assessment Methods," Comp. Gen. Pharmacol., no. March, pp. 25-26, 2003.

[34] M. Donazzan, N. Erkal, and B. H. Koh, "Impact of rebates and refunds on contributions to threshold public goods: Evidence from a field experiment," South. Econ. J., vol. 83, no. 1, pp. 69-86, 2016.

[35] J. A. List and D. Lucking-Reiley, "The effects of seed money and refunds on charitable giving: Experimental evidence from a university capital campaign," J. Polit. Econ., vol. 110, no. 1, pp. 215-233, 2002. 